\title{
Risk Associated with Cumulative Oral Glucocorticoid Use in Patients with Giant Cell Arteritis in Real-World Databases from the USA and UK
}

\author{
Sara Gale · Jessica C. Wilson · Jenny Chia · Huong Trinh • \\ Katie Tuckwell · Neil Collinson - Sophie Dimonaco · Susan Jick • \\ Christoph Meier · Shalini V. Mohan · Khaled Sarsour
}

Received: March 19, 2018 / Published online: May 11, 2018

(C) The Author(s) 2018

\section{ABSTRACT}

Introduction: Treatment of giant cell arteritis (GCA) involves immediate initiation of highdose glucocorticoid therapy with slow tapering of the dose over many months. Chronic exposure to glucocorticoids is associated with serious comorbidities. The objective of this analysis was to determine the glucocorticoid exposure and risk of glucocorticoid-related adverse events (AEs) in real-world patients with GCA.

Enhanced digital features To view enhanced digital features for this article go to https://doi.org/10.6084/ m9.figshare.6159410.

Electronic supplementary material The online version of this article (https://doi.org/10.1007/s40744018-0112-8) contains supplementary material, which is available to authorized users.

S. Gale $(\bowtie) \cdot J$. Chia $\cdot$ H. Trinh $\cdot$ K. Tuckwell ·

S. V. Mohan · K. Sarsour

Genentech, Inc., South San Francisco, CA, USA

e-mail: gale.sara@gene.com

J. C. Wilson · C. Meier

Basel Pharmacoepidemiology Unit, University of

Basel, Basel, Switzerland

N. Collinson $\cdot$ S. Dimonaco

Roche Products, Ltd., Welwyn Garden City, UK

S. Jick · C. Meier

Boston Collaborative Drug Surveillance Program, Boston University School of Public Health,

Lexington, MA, USA
Methods: Data from the Truven Healthcare MarketScan ${ }^{\circledR}$ database (from January 1, 2000, to June 30, 2015) and the Clinical Practice Research Datalink (CPRD; from January 1, 1995, to August 31, 2013) were used to retrospectively analyze patients aged $\geq 50$ years with GCA in the USA and UK, respectively. Outcomes included oral glucocorticoid use (cumulative prednisone-equivalent exposure), glucocorticoidrelated AEs and the association of AE risk with glucocorticoid exposure over 52 weeks.

Results: Of the 4804 patients in the US MarketScan database and 3973 patients in the UK CPRD database included, 71.3 and $74.6 \%$ were women and mean age was 73.4 and 73.0 years, respectively. Median starting glucocorticoid dose and cumulative glucocorticoid dose at 52 weeks were $20-50 \mathrm{mg} /$ day and 4000-4800 mg, respectively. The most frequent glucocorticoid-related AEs were hypertension and eye, bone health, and glucose tolerance conditions. In the first year after diagnosis, the likelihood of any glucocorticoid-related AE was significantly increased for each $1 \mathrm{~g}$ increase in cumulative glucocorticoid dose in the US and UK cohorts (odds ratio [95\% CI], 1.170 [1.063, 1.287] and 1.06 [1.03, 1.09], respectively; $P<0.05$ for both). Similar trends were observed for the risk of glucocorticoid-related AEs over full follow-up (mean, USA: 3.9 years, UK: 6.3 years).

Conclusions: In real-world patients with GCA, increased cumulative glucocorticoid exposure 
was associated with an increased risk of glucocorticoid-related AEs.

Funding: F. Hoffmann-La Roche Ltd.

Plain Language Summary: Plain language summary available for this article.

Keywords: Giant cell arteritis; Glucocorticoid; Safety; Toxicity; Vasculitis

\section{PLAIN LANGUAGE SUMMARY}

Patients with giant cell arteritis (GCA) are at risk of severe complications including permanent blindness. To avoid these serious complications, patients receive high doses of steroids, typically prednisone, prednisolone, or a similar glucocorticoid. The steroid dose is then reduced slowly over many months. However, relapse occurs frequently and requires additional rounds of treatment with high doses of steroids. Long-term treatment with steroids increases the risk of developing osteoporosis, infections, heart disease, diabetes, cataracts, and glaucoma. Therefore, reducing the total amount of steroids needed to treat GCA is a key challenge to improve the overall health of patients with this disease.

This study measured how the cumulative dose of steroid treatment affects the risk of steroid-related complications in patients with GCA who were treated in routine clinical practice in the United States and in the United Kingdom. Over the course of 1 year from the time of diagnosis, a patient with GCA typically received a cumulative steroid dose of $4 \mathrm{~g}$ or more. For each $1 \mathrm{~g}$ of cumulative steroid dose a patient received, that patient's risk of developing a complication related to steroid treatment increased by 3 to $8 \%$. This increase in risk underscores the need for new therapies that reduce the total amount of steroids needed for the treatment of GCA.

\section{INTRODUCTION}

Giant cell arteritis (GCA) is the most common form of systemic vasculitis, affects medium and large arteries, and is typified by histological features of localized inflammation in the adventitia and media of arterial walls and infiltrates of macrophages, T cells, and multinucleated giant cells [1-5]. GCA is rare among patients aged $<50$ years, with median age of onset of 77 years $[2,3,6]$. Women are more likely than men to develop GCA $[2,7]$. Caucasians of Northern European descent are at an increased risk of GCA $[1-3,8]$.

Classification criteria for GCA were established by the American College of Rheumatology in 1990 [9]. Markers of inflammation, including ESR and CRP, are elevated in GCA. Symptoms comprise severe headache (often of new onset), jaw and limb claudication, scalp and tongue necrosis, polymyalgia rheumatica and visual disturbances, including blurred vision, diplopia, and amaurosis fugax $[9,10]$. If untreated, vision loss can become permanent $\leq 48 \mathrm{~h}$ after onset of symptoms [11].

The standard of care for GCA involves immediate initiation of high doses of glucocorticoids to provide immediate relief of symptoms and to avoid permanent visual impairment. Treatment guidelines recommend slow tapering of glucocorticoid doses over many months after symptoms have resolved and ESR and CRP levels have normalized [12-15]. Relapse is frequent, occurring in up to $50 \%$ of patients, and requires re-initiation of the last effective glucocorticoid dose or a higher dose at the physician's discretion $[16,17]$.

Chronic glucocorticoid exposure in patients with GCA is associated with frequent occurrence of serious comorbidities, including osteoporosis, infections, cardiovascular disease, diabetes mellitus, cataracts, and glaucoma [16, 18-21]. A similar burden of comorbidities exists in patients who received glucocorticoid treatment for rheumatoid arthritis $[22,23]$. The Glucocorticoid Toxicity Index (GTI) is a recently developed tool to measure the complex burden of glucocorticoid-related comorbidities across diseases [24]. The GTI is based on a set of clinical questions, which are not captured in retrospective databases [24]. To adapt the approach of the GTI to an analysis of claims data, Medical Dictionary for Regulatory Activities groups of the GTI were translated into International Classification of Diseases, Ninth 
Revision, Clinical Modification (ICD-9-CM) and Read codes.

Mitigating the risks of glucocorticoid-related adverse events (AEs) is important, as patients with GCA tend to be older, with significant comorbidities at the time of diagnosis and treatment initiation. A quantitative understanding of the contributions of glucocorticoid exposure to risks of glucocorticoid-related AEs could inform treatment decisions and design of future clinical trials in GCA. The recently demonstrated efficacy of tocilizumab as a steroid-sparing agent in patients with GCA in the GiACTA clinical trial has created an opportunity to re-evaluate standard-of-care practices with regard to glucocorticoid therapy in this patient population [25].

In this study, glucocorticoid exposure and glucocorticoid-related AEs were examined in two distinct real-world cohorts of patients with GCA from US and UK healthcare claims databases. These data provide a representation of current treatment practices and potential adverse consequences of glucocorticoid therapy. The objectives of this study were to describe real-world use of oral glucocorticoids and to estimate the risk of glucocorticoid-related AEs adapted from the GTI and associated with cumulative glucocorticoid exposure over time in patients with GCA.

\section{METHODS}

\section{Data Sources}

The Truven Healthcare MarketScan ${ }^{\circledR}$ Commercial Claims and Encounters (Commercial) database and Medicare Supplemental and Coordination of Benefits database (Medicare) contain claims data for $\sim 65$ million US patients, aged $<65$ years with private-sector insurance plans or aged $\geq 65$ years with Medicare and employer-sponsored supplemental insurance.

The Clinical Practice Research Datalink (CPRD) contains data for $\sim 12$ million patients; these data are broadly representative of the UK general population in terms of age, sex, body mass index, and ethnicity [26]. The CPRD includes information about visits to general practitioners (GPs) and demographics, outcomes, diagnoses, prescriptions, and referrals recorded by GPs. The study protocol was reviewed and approved by the Independent Scientific Advisory Committee for Medicines and Healthcare Products Regulatory Agency. Additional data for the UK cohort were obtained from Hospital Episode Statistics, which contains details of all patient admissions to National Health Service hospitals in England, including $\sim 55 \%$ of patients in the CPRD.

This study is based on previously conducted studies and does not contain any studies with human participants or animals performed by any of the authors. This study used only deidentified patient data; therefore, informed consent for this study was not needed.

\section{Study Design}

This study comprised retrospective analyses of glucocorticoid use and AEs in two cohorts of patients with GCA in the United States and United Kingdom. Patients were followed for $\geq 1$ year after claims or GP-recorded data indicated a GCA diagnosis (Fig. 1). Glucocorticoid prescriptions, glucocorticoid-related AEs, and adverse events of special interest (AESIs) were identified by code and included in the analyses.

\section{Patient Populations}

Patients with GCA who received care from January 1,2000 , to June 30, 2015, were identified in the MarketScan database and included in the US cohort. Inclusion criteria were $\geq 1$ inpatient claim or $\geq 2$ outpatient claims separated by $\geq 7$ days and $\leq 365$ days with confirmatory GCA listed (excluding diagnostic GCA claims) among diagnoses (ICD-9-CM code $446.5) ; \geq 2$ glucocorticoid prescriptions, with the first $\leq 6$ months after index (index date was defined as the first date of GCA diagnosis) and the second $\leq 6$ months after the first (National Coverage Determination [NCD]/Healthcare Common Procedure Coding System [HCPCS] codes list available on request); age $\geq 50$ years 


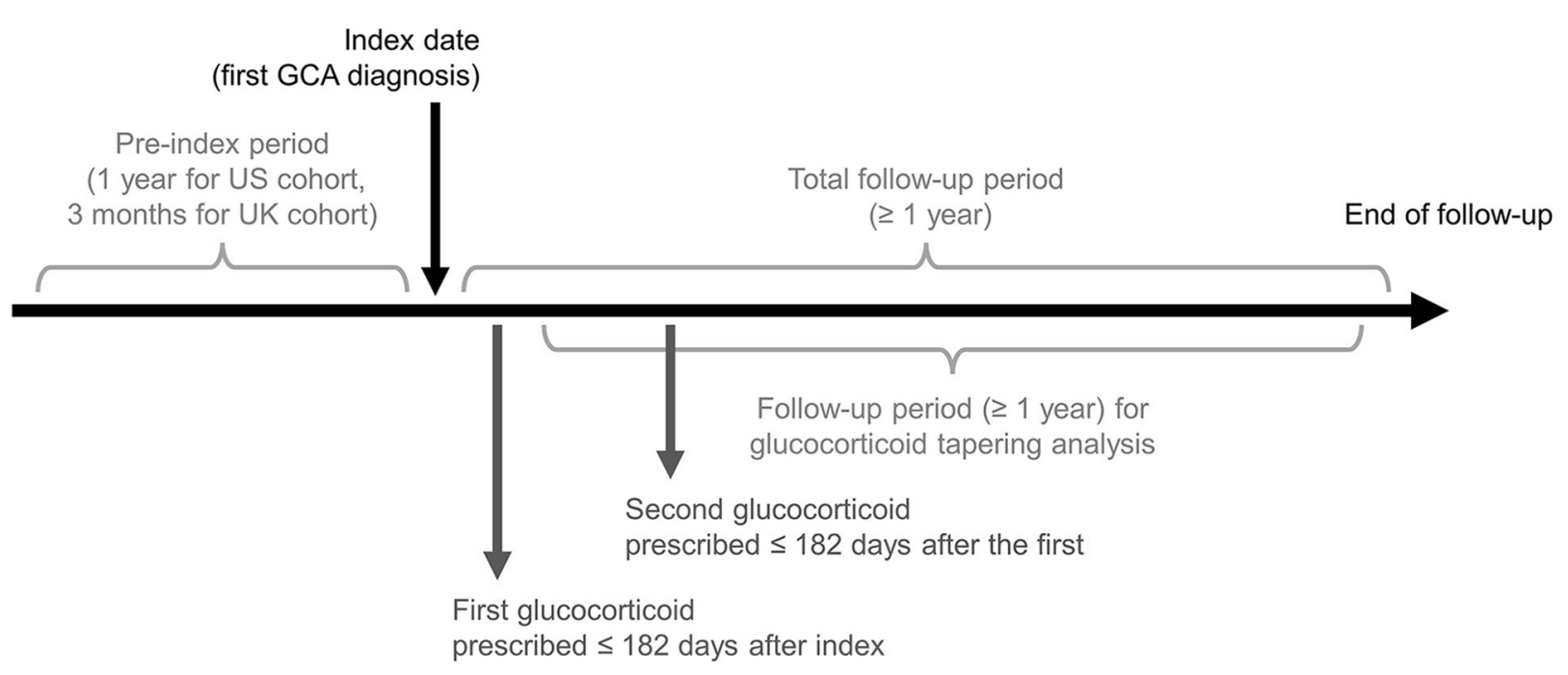

Fig. 1 Study time frame for patients with GCA in US and UK cohorts

at index; evidence of receiving $\geq 1$ diagnostic workup for temporal artery biopsy, magnetic resonance angiography, computed tomography angiography or positron emission tomography computed tomography $\leq 1$ year pre- or postindex (Current Procedural Terminology [CPT]/ HCPCS codes list available on request); no confirmatory GCA claims $\leq 1$ year prior to first GCA diagnosis to ensure that the case represents a new GCA diagnosis; $\geq 1$ GCA claim after the diagnostic workup; continuous medical and pharmacy coverage and full data available for the duration of the patient's eligibility $(\leq 30$ day gaps permitted pre-index); $\geq 365$ days of eligibility prior to first GCA diagnosis; no tocilizumab exposure; $\geq 365$ days of follow-up after index date; and $\geq 365$ days of follow-up after first glucocorticoid prescription.

Patients with GCA who received care from January 1, 1995, to August 31, 2013, in the CPRD were identified and included in the UK cohort. Inclusion criteria were aged $\geq 50$ years; diagnosis code for GCA (Read codes G755000, G755.00, G755z00, N200.00, G755100, and RG755200); $\geq 2$ prednisolone prescriptions, with the first $\leq 6$ months after GCA diagnosis and the second $\leq 6$ months after the first (Read codes list available on request); $\geq 3$ years of recorded medical history prior to first GCA diagnosis; $\geq 365$ days of follow-up after first prednisolone prescription; and no diagnosis of cancer, alcoholism, drug abuse or HIV prior to the prednisolone start date. Linkage to Hospital Episode Statistics, covering 1997 to 2012, was obtained for patients identified in the UK cohort. The years of follow-up mostly overlap between the US and UK cohorts but are not the same due to differences in access to the data at the time of the analysis.

\section{Glucocorticoid Exposure}

Oral glucocorticoid exposure was captured using the mean prednisone-equivalent dose (Supplemental Table 1). The start date for glucocorticoid exposure was the first date of glucocorticoid use after index. To describe realworld glucocorticoid use, cumulative glucocorticoid exposure was determined at 52 weeks and at the end of the study period using all available follow-up data. Tablet strength (i.e., $10 \mathrm{mg}$ or $5 \mathrm{mg}$ ) and prescription quantity were summed across all glucocorticoid prescriptions (by prednisone-equivalent dose). The most frequently used prescription quantity in the cohort was used for missing values. The duration of each oral glucocorticoid prescription was calculated by combining the dosing instructions and the number of tablets prescribed. One tablet per day was assumed for missing values. For overlaps in 
repeat prescriptions, the total duration was extended by the number of days of overlap. For instances of two glucocorticoid prescriptions on the same date, the longer of the two prescription durations was used; these were likely prescribed to be taken together to create a higher dose. For the UK cohort, only prednisolone prescriptions were included.

\section{Safety Endpoints}

Safety endpoints included glucocorticoid-related AEs classified based on the GTI and AESIs, which were AEs observed during the tocilizumab clinical trial program [24]. Additionally, specific AEs from the glucocorticoid-related groupings and the AESI list were evaluated. For glucocorticoid-related AEs and AESIs, patients were excluded if the $\mathrm{AE}$ occurred $\leq 30$ days or $\leq 365$ days prior to index, depending on the AE type (Supplemental Table 2). Glucocorticoid-related AEs comprised diagnosis codes for blood pressure, endocrine, bone-related, muscle and tendon, eye, glucose tolerance, gastrointestinal tract, skin, and neuropsychiatric conditions [24]. AESIs to tocilizumab comprised myocardial infarction, ischemic stroke, hemorrhagic stroke, cerebrovascular accident, acute coronary syndrome, heart failure, acute hepatic failure, hepatic transplant, hepatotoxic events, gastrointestinal perforation, malignancies, serious infections, opportunistic infections, pneumonia, serious gastrointestinal bleeding events, and demyelination. Patients were classified as having experienced an AESI if hospitalization ( $\geq$ 1-day hospital stay) occurred with a diagnosis code indicative of the AESI on or after index. The exceptions were malignancies, which required $\geq 1$ inpatient or $\geq 2$ outpatient claims (separated by $\geq 7$ days), and serious infections, which required either hospitalization or $\geq 1$ outpatient claim requiring intravenous antibiotics ( $\leq 7$ days prior to or after infection diagnosis) [27].

\section{Statistical Analysis}

Two approaches were used to determine the association between glucocorticoid use over time and the risk of an AE: a logistic regression model and a Cox proportional hazards model. Both models estimated the risk of developing an $\mathrm{AE}$ associated with a 1-g increase in cumulative glucocorticoid exposure over time, where cumulative glucocorticoid exposure was modeled as a continuous variable and cumulative prednisone-equivalent exposure (in grams) was used to index glucocorticoid exposure. The logistic regression model examined the risk of an $\mathrm{AE}$ over the first 52 weeks from the index date. In the logistic regression model, the glucocorticoid exposure variable was modeled as the cumulative glucocorticoid dose. The Cox model evaluated the long-term risk of an $\mathrm{AE}$ over the full follow-up duration. The full followup duration could vary from patient to patient, with a minimum duration of 52 weeks. In the Cox model, the glucocorticoid exposure variable was modeled as a combination of the daily dose and the cumulative dose of glucocorticoids. Both models were adjusted for age, sex, baseline comorbidities (Charlson Comorbidity Index), previous disease history (occurrence of the event 30 days or 365 days prior to index) and glucocorticoid exposure as independent variables.

For the individual glucocorticoid-related $\mathrm{AE}$ analyses (e.g., pneumonia, fracture), patients without that specific AE were censored at the end of follow-up. Patients with the specific AE in the 30 days or 365 days prior to index were excluded from the model. Adjusted hazard ratios and 95\% CIs are reported.

When incidence rates were generated and risk models were run, only those patients who had the AE under investigation were excluded, not the total number of patients who had any of the baseline exclusionary AEs.

In the GiACTA study, the mean cumulative prednisone dose was 2097.8 and $2447.0 \mathrm{mg}$ in the tocilizumab weekly and every-other-week dose groups, respectively; patients in both groups received tocilizumab in combination with a 26-week prednisone taper [25]. These mean cumulative prednisone doses were applied as cutoffs to the real-world data glucocorticoid doses to categorize patients as having either high or low glucocorticoid exposure. The absolute risk difference between high and low 
Table 1 Baseline demographics for patients with GCA by study cohort

\begin{tabular}{|c|c|c|}
\hline Characteristic & $\begin{array}{l}\text { US cohort }\left(\text { MarketScan }{ }^{\circledR} 2000-2015\right) \\
(N=4804)\end{array}$ & $\begin{array}{l}\text { UK cohort }(\text { CPRD 1995-2013) } \\
(N=3973)\end{array}$ \\
\hline Female $(n(\%))$ & $3425(71.3)$ & $2964(74.6)$ \\
\hline $\begin{array}{l}\text { Age at index (mean }(\mathrm{SD}) \\
(\text { years }))\end{array}$ & $73.4(9.8)$ & $73.0(8.9)$ \\
\hline \multicolumn{3}{|l|}{ Age group (n (\%) (years)) } \\
\hline $50-54$ & $145(3.0)$ & $109(2.7)$ \\
\hline $55-59$ & $338(7.0)$ & $259(6.5)$ \\
\hline $60-64$ & $613(12.8)$ & $372(9.4)$ \\
\hline $65-69$ & $484(10.1)$ & $580(14.6)$ \\
\hline $70-74$ & $786(16.4)$ & $855(21.5)$ \\
\hline $75-79$ & $985(20.5)$ & $849(21.4)$ \\
\hline $80-84$ & $872(18.2)$ & $597(15.0)$ \\
\hline$>84$ & $581(12.1)$ & $352(8.9)$ \\
\hline \multicolumn{3}{|l|}{ Smoking status, $n$ (\%) } \\
\hline Never & NA & $1933(48.7)$ \\
\hline Current & NA & $621(15.6)$ \\
\hline Former & NA & $1148(28.9)$ \\
\hline Missing & NA & $271(6.8)$ \\
\hline \multicolumn{3}{|c|}{ Body mass index $\left(n(\%)\left(\mathrm{kg} / \mathrm{m}^{2}\right)\right)$} \\
\hline $12-18.4$ & NA & $75(1.9)$ \\
\hline $18.5-24.9$ & NA & $1283(32.3)$ \\
\hline $25-29.9$ & NA & $1326(33.3)$ \\
\hline $30-60$ & NA & $694(17.5)$ \\
\hline Missing & NA & $595(15.0)$ \\
\hline \multicolumn{3}{|l|}{ Alcohol use } \\
\hline Never & NA & $979(24.6)$ \\
\hline Current & NA & $2463(62.0)$ \\
\hline Former & NA & $66(1.7)$ \\
\hline Missing & NA & $465(11.7)$ \\
\hline
\end{tabular}

$C P R D$ Clinical Practice Research Datalink, $N A$ not available

glucocorticoid exposure groups was calculated for the risk of experiencing any glucocorticoidrelated $\mathrm{AE}$ within 52 weeks. The risk difference was calculated using a generalized linear model with a binomial distribution and the identity link. 


\section{RESULTS}

\section{Baseline Patient Demographics}

The demographic characteristics of both the US cohort $(N=4804)$ and UK cohort $(N=3973)$ were typical for patients with GCA (Table 1). Patients were predominately female $(>70 \%)$ and elderly, with mean age of $\sim 73$ years. Among UK patients, $50.8 \%$ were overweight or obese (body mass index $>25 \mathrm{~kg} / \mathrm{m}^{2}$ ), $15.6 \%$ were current and $28.9 \%$ were former smokers, and $62.0 \%$ consumed alcohol. Data on these characteristics were not available for US patients.

\section{Glucocorticoid Use}

The median (interquartile range [IQR]) starting daily dose of oral glucocorticoids among all patients in the US cohort was $50(25-60) \mathrm{mg}$, based on prednisone-equivalent dose (Table 2). Among the subgroup of UK patients who had complete daily dose instructions with their prescription fills $(n=278)$, the median (IQR) starting daily dose of oral glucocorticoids was 20 (10-40) mg. The median (IQR) cumulative dose of oral glucocorticoids at 52 weeks from the first glucocorticoid dose in the US $(n=4804)$ and UK $(n=3973)$ cohorts was $4800(2700-7317) \mathrm{mg}$ and 4000 (2380-5930) $\mathrm{mg}$, respectively.

\section{Risk of Adverse Events}

A logistic regression model indicated a statistically significant increase in the likelihood of US patients experiencing any glucocorticoid-related $\mathrm{AE}$ associated with each $1-\mathrm{g}$ increase in cumulative glucocorticoid dose in the first year following a GCA diagnosis (odds ratio [95\% CI], 1.170 [1.063, 1.287]; Table 3). Statistically significant increases in the likelihood of developing a bone-, musculoskeletal-, eye-, glucose tolerance-, or skin-related disorder were found in the US cohort for each $1-\mathrm{g}$ increase in cumulative glucocorticoid dose over 52 weeks. The likelihood of US patients experiencing an AESI to tocilizumab (odds ratio [95\% CI], 1.056 $[1.008,1.105])$ significantly increased with each 1-g increase in cumulative glucocorticoid dose in the first year following diagnosis. The likelihood of fractures, glaucoma, and serious infections in the US cohort were also significantly increased for each 1-g increase in cumulative glucocorticoid dose. No clear differences were found in the likelihood of a glucocorticoid-related $\mathrm{AE}$ or an AESI over 52 weeks between patients of different age groups.

In the UK cohort, significant increases were found in the overall likelihood of developing any glucocorticoid-related $\mathrm{AE}$ and the likelihood of musculoskeletal-, eye-, glucose tolerance- or skin-related disorders with each 1-g increase in cumulative glucocorticoid exposure over the first years following a GCA diagnosis. The likelihood of diabetes, glaucoma, cataracts, and serious infections was also significantly increased for each 1-g increase in cumulative glucocorticoid dose, but the likelihood of experiencing an AESI to tocilizumab was not increased (Table 3).

Over the full follow-up in the US (mean, 3.9 years) and UK cohorts (mean, 6.3 years), the Cox proportional hazards model indicated that the risk of a glucocorticoid-related $\mathrm{AE}$ was significantly increased with each 1-g increase in cumulative glucocorticoid exposure (Table 4). In both cohorts, statistically significant increased risks were associated with each 1-g increase in cumulative glucocorticoid exposure for the glucocorticoid-related AE categories of endocrine, bone-related, eye, glucose tolerance, and skin conditions; for the specific AEs of anemia, cataracts, diabetes, osteoporosis, and serious infections; and for AESIs to tocilizumab. Statistically significant increased risks were found for blood pressure and neuropsychiatric conditions in the US cohort (but not the UK cohort) and for musculoskeletal-related conditions in the UK cohort (but not the US cohort).

In the US cohort, a 9\% risk reduction was observed for a glucocorticoid-related $\mathrm{AE}$ in the first year following a GCA diagnosis for patients who received a cumulative glucocorticoid dose that was identical to or lower than the doses received by the tocilizumab groups in the GiACTA study (Table 5). In the UK cohort, a 6\% risk reduction was observed for a glucocorticoid-related AE in the first year following a GCA 
Table 2 Summary of glucocorticoid use in patients with GCA by study cohort

\begin{tabular}{|c|c|c|}
\hline Glucocorticoid use & $\begin{array}{l}\text { US cohort } \\
(N=4804)\end{array}$ & $\begin{array}{l}\text { UK cohort } \\
(N=3973)^{\mathrm{a}}\end{array}$ \\
\hline Daily starting dose of first GC after index & $(n=4804)$ & $(n=278)^{\mathrm{b}}$ \\
\hline Mean (SD) (mg) & $47(35)$ & $24(17)$ \\
\hline Median (IQR) (mg) & $50(25-60)$ & $20(10-40)$ \\
\hline Range (mg) & $0.11-800$ & $1-100$ \\
\hline Cumulative GC dose 52 weeks after index & $(n=4804)$ & $(n=3973)$ \\
\hline Mean (SD) (mg) & $5577(4985)$ & $4432(2792)$ \\
\hline Median (IQR) (mg) & $4800(2700-7317)$ & $4000(2380-5930)$ \\
\hline Range (mg) & $10-140,600$ & $48-28,050$ \\
\hline Patients on $0 \mathrm{mg} /$ day at 52 weeks $(n(\%))^{\mathrm{c}}$ & $1810(47)$ & $1631(41.1)$ \\
\hline Cumulative GC dose from index to end of study & $(n=4804)$ & $(n=3973)$ \\
\hline Mean (SD) (mg) & $8685.9(8500.7)$ & $9620.3(8497.5)$ \\
\hline Median (IQR) (mg) & $6750(3600-11,173)$ & $7233(3790-13,025)$ \\
\hline Range (mg) & $10-156,200$ & $61-77,225$ \\
\hline $\begin{array}{l}\text { Total supply of GC in days from index to end of follow-up (mean } \\
\text { (median)) }\end{array}$ & $485(338)$ & $1405(1175)$ \\
\hline Patients with GC fills after 52 weeks $(n(\%))$ & $3650(76)$ & $3185(80)$ \\
\hline Among patients continuing to receive GC after 52 weeks & $(n=3650)$ & $(n=3185)$ \\
\hline Length of treatment from index to end of follow-up (mean (SD) (days)) & $600(510)$ & $1660(1170)$ \\
\hline Median (IQR) (days) & $441(282-749)$ & $1324(775-2247)$ \\
\hline Range (days) & $17-4309$ & $18-6748$ \\
\hline
\end{tabular}

$G C$ glucocorticoid, $I Q R$ interquartile range

a Only prednisolone prescriptions were included in the analysis of UK patients; $1.2 \%$ of UK patients had prescriptions for other glucocorticoids (methylprednisolone was prescribed for $0.05 \%$ )

b Only patients with complete information on dosage instructions were included in the starting dose calculation

c Only patients who had a fill during week 26 or 56 were included; patients could be excluded if they tapered to $0 \mathrm{mg} / \mathrm{day}$ or were still on glucocorticoids but their prescription fill was not during week 26 or week 52

diagnosis using the same cutoffs for cumulative glucocorticoid dose.

\section{DISCUSSION}

The real-world US and UK cohorts in this study had demographic characteristics typical of patients with GCA $[16,18,28,29]$. They were exposed to substantial cumulative doses of glucocorticoids in real-world clinical practice settings in the first year following GCA diagnosis (median, 4-4.8 g) and throughout their disease course (median, 6.8-7.2 g). Patients had a significantly greater risk of developing glucocorticoid-related AEs for each additional $1 \mathrm{~g}$ of cumulative glucocorticoid exposure both over the first year following diagnosis and over the full follow-up. In both cohorts, increased risk was found for diabetes (3-4.9\%), fractures 
Table 3 Odds ratios for various SAEs for each 1-g increase in cumulative glucocorticoid use over 52 weeks by study cohort

\begin{tabular}{|c|c|c|}
\hline \multirow[t]{2}{*}{ Serious adverse events } & \multicolumn{2}{|l|}{ Odds ratio $(95 \% \mathrm{CI})^{\mathrm{a}}$} \\
\hline & $\overline{\text { US } \text { cohort }(N=4804)}$ & UK cohort $(N=3973)$ \\
\hline \multicolumn{3}{|l|}{ GC-related AEs ${ }^{\mathrm{b}}$} \\
\hline Any GC-related event & $1.17(1.06,1.29)^{\mathrm{c}}$ & $1.06(1.03,1.09)^{\mathrm{c}}$ \\
\hline Blood pressure & $1.04(1.00,1.09)$ & $1.06(1.00,1.08)$ \\
\hline Endocrine & $1.12(0.98,1.28)$ & $1.04(0.75,1.44)$ \\
\hline Bone-related conditions & $1.07(1.03,1.12)^{\mathrm{c}}$ & $1.03(0.99,1.06)$ \\
\hline Muscle and tendon & $1.11(1.01,1.21)^{\mathrm{c}}$ & $1.15(1.05,1.27)^{\mathrm{c}}$ \\
\hline Eye & $1.06(1.01,1.11)^{\mathrm{c}}$ & $1.12(1.07,1.17)^{\mathrm{c}}$ \\
\hline Glucose tolerance & $1.14(1.09,1.20)^{\mathrm{c}}$ & $1.07(1.02,1.11)^{\mathrm{c}}$ \\
\hline Gastrointestinal tract & $1.00(0.81,1.25)$ & $0.89(0.76,1.04)$ \\
\hline Skin & $1.10(1.03,1.17)^{\mathrm{c}}$ & $1.09(1.03,1.15)^{\mathrm{c}}$ \\
\hline Neuropsychiatric & $1.05(0.98,1.12)$ & $1.04(0.99,1.09)$ \\
\hline \multicolumn{3}{|l|}{ Other SAEs } \\
\hline Adrenal insufficiency & $1.12(0.98,1.28)$ & $1.04(0.72,1.51)$ \\
\hline Anemia & $1.04(0.99,1.10)$ & $1.02(0.96,1.08)$ \\
\hline Cataracts & $1.02(0.97,1.08)$ & $1.09(1.03,1.16)^{\mathrm{c}}$ \\
\hline Cerebrovascular disease & $1.01(0.93,1.10)$ & $1.01(0.95,1.07)$ \\
\hline Depression & $1.06(0.97,1.15)$ & $0.98(0.91,1.06)$ \\
\hline Diabetes & $1.12(1.06,1.18)^{\mathrm{c}}$ & $1.12(1.06,1.17)^{\mathrm{c}}$ \\
\hline Fracture & $1.09(1.04,1.15)^{\mathrm{c}}$ & $1.02(0.94,1.10)$ \\
\hline Gastrointestinal perforation & $1.00(0.80,1.27)$ & $1.01(0.71,1.43)$ \\
\hline Glaucoma & $1.09(1.01,1.17)^{\mathrm{c}}$ & $1.14(1.09,1.21)^{\mathrm{c}}$ \\
\hline Liver disease & $1.16(0.92,1.46)$ & $\mathrm{NA}$ \\
\hline Myocardial infarction & $0.96(0.86,1.08)$ & $0.87(0.77,0.99)^{\mathrm{c}}$ \\
\hline Osteoporosis & $1.06(1.00,1.13)^{\mathrm{c}}$ & $1.03(0.98,1.09)$ \\
\hline Serious infections & $1.05(1.01,1.10)^{\mathrm{c}}$ & $1.06(1.00,1.12)^{\mathrm{c}}$ \\
\hline Any AESI to tocilizumab & $1.06(1.01,1.10)^{c}$ & $0.99(0.95,1.03)$ \\
\hline
\end{tabular}

$A E$ adverse event, $A E S I$ adverse event of special interest, $G C$ glucocorticoid, $N A$ not available, $S A E$ serious adverse event

${ }^{a}$ Regression adjusted for covariates of age, sex, Charlson Comorbidity Index, and previous disease history

b Classes of AEs included in the evaluation of the Glucocorticoid Toxicity Index [24]

${ }^{c} P<0.05$

(1-1.9\%), glaucoma (2-2.9\%), osteoporosis (3-3.4\%) and serious infections (2-3.2\%) for each 1-g increase in cumulative glucocorticoid exposure over the full follow-up. Furthermore, patients with cumulative glucocorticoid exposure greater than the mean cumulative 
Table 4 Hazard ratios for various SAEs for each 1-g increase in cumulative glucocorticoid use over full follow-up by study cohort

\begin{tabular}{|c|c|c|}
\hline \multirow[t]{2}{*}{ Serious adverse events } & \multicolumn{2}{|l|}{ Hazard ratio $(95 \% \mathrm{CI})^{\mathrm{a}}$} \\
\hline & US cohort $(N=4804)^{b}$ & UK cohort $(N=3973)^{c}$ \\
\hline \multicolumn{3}{|l|}{ GC-related AEs ${ }^{\mathrm{d}}$} \\
\hline Any GC-related event & $1.08(1.00,1.16)^{\mathrm{e}}$ & $1.03(1.02,1.04)^{\mathrm{e}}$ \\
\hline Blood pressure & $1.04(1.01,1.07)^{\mathrm{e}}$ & $1.00(0.98,1.01)$ \\
\hline Endocrine & $1.05(1.02,1.09)^{\mathrm{e}}$ & $1.09(1.01,1.17)^{\mathrm{e}}$ \\
\hline Bone-related conditions & $1.03(1.01,1.04)^{\mathrm{e}}$ & $1.02(1.01,1.03)^{\mathrm{e}}$ \\
\hline Muscle and tendon & $1.04(1.00,1.07)$ & $1.06(1.03,1.10)^{\mathrm{e}}$ \\
\hline Eye & $1.04(1.02,1.06)^{\mathrm{e}}$ & $1.04(1.03,1.05)^{\mathrm{e}}$ \\
\hline Glucose tolerance & $1.05(1.03,1.06)^{\mathrm{e}}$ & $1.02(1.01,1.04)^{\mathrm{e}}$ \\
\hline Gastrointestinal tract & $1.00(0.91,1.10)$ & $1.02(0.99,1.06)$ \\
\hline Skin & $1.04(1.02,1.05)^{\mathrm{e}}$ & $1.03(1.01,1.04)^{\mathrm{e}}$ \\
\hline Neuropsychiatric & $1.03(1.01,1.05)^{\mathrm{e}}$ & $1.01(0.99,1.02)$ \\
\hline \multicolumn{3}{|l|}{ Other SAEs } \\
\hline Adrenal insufficiency & $1.05(1.02,1.09)^{\mathrm{e}}$ & $1.00(0.81,1.24)$ \\
\hline Anemia & $1.02(1.01,1.04)^{\mathrm{e}}$ & $1.02(1.01,1.04)^{\mathrm{e}}$ \\
\hline Cataracts & $1.03(1.01,1.05)^{\mathrm{e}}$ & $1.04(1.03,1.05)^{\mathrm{e}}$ \\
\hline Cerebrovascular disease & $1.01(0.98,1.04)$ & $1.00(0.99,1.02)$ \\
\hline Depression & $1.02(1.00,1.05)^{\mathrm{e}}$ & $1.01(1.00,1.03)$ \\
\hline Diabetes & $1.05(1.03,1.07)^{\mathrm{e}}$ & $1.03(1.01,1.05)^{\mathrm{e}}$ \\
\hline Fracture & $1.02(1.00,1.04)^{\mathrm{e}}$ & $1.01(1.00,1.03)$ \\
\hline Gastrointestinal perforation & $1.04(0.98,1.09)$ & $1.09(0.99,1.20)$ \\
\hline Glaucoma & $1.03(0.98,1.08)$ & $1.02(1.00,1.05)$ \\
\hline Liver disease & $1.03(0.95,1.12)$ & NA \\
\hline Myocardial infarction & $1.01(0.99,1.04)$ & $1.01(0.99,1.03)$ \\
\hline Osteoporosis & $1.03(1.02,1.05)^{\mathrm{e}}$ & $1.03(1.01,1.04)^{\mathrm{e}}$ \\
\hline Serious infections & $1.03(1.02,1.04)^{\mathrm{e}}$ & $1.02(1.01,1.04)^{\mathrm{e}}$ \\
\hline Any AESI to tocilizumab & $1.02(1.01,1.04)^{\mathrm{e}}$ & $1.01(1.01,1.02)^{\mathrm{e}}$ \\
\hline
\end{tabular}

$A E$ adverse event, $A E S I$ adverse event of special interest, $G C$ glucocorticoid, $N A$ not available, $S A E$ serious adverse event

${ }^{a}$ Regression adjusted for covariates of age, sex, Charlson Comorbidity Index, and previous disease history

b Mean (median) follow-up duration for the US cohort was 3.9 (3.1) years

${ }^{c}$ Mean (median) follow-up duration for the UK cohort was 6.3 (5.5) years

d Classes of AEs included in the evaluation of the Glucocorticoid Toxicity Index [24]

e $P<0.05$ 
Table 5 Risk differences between high and low glucocorticoid exposure (compared with glucocorticoid exposure in the GiACTA study) of any SAE over 52 weeks by study cohort

\begin{tabular}{lll}
\hline Exposure type & \multicolumn{2}{l}{$\begin{array}{l}\text { Risk difference by cutoff for high vs. } \\
\text { low GC exposure }(\mathbf{9 5 \%} \mathbf{C I})^{\mathbf{a}}\end{array}$} \\
\cline { 2 - 3 } & $\begin{array}{l}\text { US cohort } \\
(\boldsymbol{N}=\mathbf{4 8 0 4})\end{array}$ & $\begin{array}{l}\text { UK cohort } \\
(\boldsymbol{N}=\mathbf{3 9 7 3})\end{array}$ \\
\hline $\begin{array}{l}\text { Cutoff based on mean cumulative GC exposure in patients who received } \\
\text { tocilizumab weekly (\%) }\end{array}$ & $9.25(4.73,13.77)^{\mathrm{b}}$ & $6.05(1.67,10.43)^{\mathrm{b}}$ \\
$\begin{array}{l}\text { Cutoff based on mean cumulative GC exposure in patients who received } \\
\text { tocilizumab every other week (\%) }\end{array}$ & $9.22(4.73,13.72)^{\mathrm{b}}$ & $6.19(2.11,10.27)^{\mathrm{b}}$ \\
\hline
\end{tabular}

$G C$ glucocorticoid, $S A E$ serious adverse event

${ }^{a}$ High GC exposure: cumulative GC dose $>$ mean cumulative GC dose in patients who received tocilizumab in the GiACTA study. Low GC exposure: cumulative GC dose $\leq$ mean cumulative GC dose in patients who received tocilizumab in the GiACTA study. Regression adjusted for covariates of age, sex, Charlson Comorbidity Index, and previous disease history

b $P<0.05$

glucocorticoid doses (2097.8 $\mathrm{mg}$ or $2447.0 \mathrm{mg}$ ) in patients who received tocilizumab in GiACTA had significantly greater risk of developing a glucocorticoid-related $\mathrm{AE}$ over 52 weeks (6-9\% increased risk) than those exposed to lower cumulative doses. This provides an estimate of the expected reduction in risk of glucocorticoid-related AEs in real-world patients with GCA if their cumulative glucocorticoid exposure were to be reduced with tocilizumab treatment.

The increased risk of developing glucocorticoid-related AEs was consistent with previous findings in UK patients with GCA [19, 20]. Trends were similar despite the smaller size of the cohort and differences in outcome definitions in this analysis. Also consistent with previous findings, a significant increase with cumulative glucocorticoid exposure was observed for diabetes, osteoporosis, and serious infections $[19,20]$.

The median starting dose of glucocorticoids in the US cohort ( $50 \mathrm{mg}$ /day) was similar to the median starting doses previously described in an observational cohort of US patients with GCA (60 mg/day) and in a study based on MarketScan claims data that used a different definition of GCA (40 mg/day) [16, 30]. The high starting doses of glucocorticoids were also consistent with treatment guidelines for GCA (recommended starting dose, $40-60 \mathrm{mg} /$ day or $\geq 60 \mathrm{mg} /$ day when visual complications are present) [13-15]. Over the full follow-up (mean, 3.9 and 6.3 years in US and UK cohorts, respectively), patients with GCA had cumulative glucocorticoid doses (mean [SD], 8.7 [8.5] g and $9.6[8.5] \mathrm{g}$ ) consistent with findings that $30 \%$ of UK patients with GCA received $\geq 10 \mathrm{~g}$ of oral glucocorticoids during the course of their disease [18]. In the UK cohort, the median starting dose was lower compared with the US cohort (20 vs. $50 \mathrm{mg} /$ day), and lower than expected based on published European and British treatment recommendations [1, 14]. Only a small subgroup of UK patients $(<10 \%)$ had daily dose instructions available for analysis; this subgroup may not be representative of the full cohort. The cumulative doses over the course of 1 year, however, were similar (4.0 vs. $4.8 \mathrm{~g})$.

The consistent elevation in risk of developing a glucocorticoid-related AE in this study was approximately $3-5 \%$ per additional $1 \mathrm{~g}$ of exposure over the full follow-up. Although the absolute risk related to glucocorticoid exposure appears low, the multiple-gram cumulative exposure in individual patients over the duration of their disease course makes the risk of experiencing a glucocorticoid-related AE substantial. In a previously studied US cohort, $86 \%$ patients with GCA developed $\geq 1$ glucocorticoid-related serious AE [16]. The population- 
level impact of this risk makes glucocorticoidrelated AEs a significant public health burden. Furthermore, the risk of developing a glucocorticoid-related AE may not be a linear function of cumulative dose.

The increased risk of serious infections observed in this study is consistent with a recent prospective study in France, which found an elevated risk of serious infections and infection-related deaths during the first year after GCA diagnosis in older patients and in patients with diabetes or with long-term high-dose glucocorticoid exposure [31]. In a study of The Health Improvement Network, a general practice database similar to the CPRD, serious infection incidence rates were significantly higher in patients with GCA compared with the general population [19].

This study has three key strengths. First, each patient cohort was $>10$-fold larger (3973 and 4804 vs. 251 patients) and had less restrictive inclusion criteria than in the GiACTA study, which excluded patients with many comorbidities that are relevant in those $\geq 50$ years of age, including malignancies, active or recent infections, immunodeficiency, liver disease and uncontrolled cardiovascular or respiratory disease [21]. Second, the patients in these cohorts received glucocorticoid doses according to the treating physician's discretion without the rigorously defined steroid tapering schedule and escape criteria used in GiACTA. Third, these analyses evaluate glucocorticoid-related safety outcomes in two cohorts of patients with a rare disease from large databases with long followup time to complement the shorter and smaller pivotal clinical trial.

This study also has several limitations. The GCA population comprises patients who have a severe disease burden and are primarily elderly; these features themselves increase the risk of AEs. Per standard of care, all patients with GCA receive glucocorticoids, so no comparisons can be made with a cohort of patients without glucocorticoid use. Claims data provide an incomplete picture for health research, as most clinical characteristics are not typically collected, few patient demographic variables are captured (increasing confounding with respect to basic characteristics in risk analyses) and outcomes important for reimbursement are likely to be overrepresented due to the databases' purposes in medical billing (not healthcare research). The MarketScan data are a nonrandom sample of patients, representing patients with access to medium to large employer-sponsored insurance plans and those who can afford to purchase commercial insurance. The CPRD lacks information on specialist and hospital visits, and linkage to other data sources is imperfect; thus care provided in hospitals or by a non-participating physician will be missed. Additionally, the CPRD has high positive predictive value but potentially low sensitivity of a disease measure, potentially biasing incidence rates toward underestimation, and codes used in the CPRD lack standardized disease definitions [26]. Both data sets have differences in underlying definitions, and underreporting in the general practice setting in the UK is likely due to data collection methods. Finally, neither data set can convey causality (e.g., if a patient is receiving another medication known to cause $\geq 1 \mathrm{AE}$ classified as a glucocorticoid-related $\mathrm{AE}$ ).

\section{CONCLUSIONS}

This study measured the cumulative glucocorticoid dose in real-world patients with GCA and estimated a 3-8\% increased risk of glucocorticoid-related AEs in these patients for each 1-g increase in cumulative glucocorticoid exposure. Further quantitative investigation is needed to understand how the cumulative glucocorticoid exposure correlates with the cumulative risk of glucocorticoid-related AEs. These findings highlight the need for widespread use of steroid-sparing therapy to prevent relapse and to accelerate glucocorticoid tapering in patients with GCA.

\section{ACKNOWLEDGEMENTS}

Funding. This work was supported by F. Hoffmann-La Roche Ltd and Genentech, Inc. Article processing charges were funded by $\mathrm{F}$. 
Hoffmann-La Roche Ltd. All authors had full access to all of the data in this study and take complete responsibility for the integrity of the data and accuracy of the data analysis.

Authorship. All named authors meet the International Committee of Medical Journal Editors (ICMJE) criteria for authorship for this article, take responsibility for the integrity of the work as a whole, and have given their approval for this version to be published.

Medical Writing and/or Editorial Assistance. Support for third-party writing assistance for this manuscript, furnished by Benjamin L. Ricca, PhD, of Health Interactions, was provided by F. Hoffmann-La Roche, Ltd.

Disclosures. S. Gale is an employee of Genentech, Inc. J. Chia is an employee of Genentech, Inc. H. Trinh is an employee of Genentech, Inc. K. Tuckwell is an employee of Genentech, Inc. S. Mohan is an employee of Genentech, Inc. K. Sarsour is an employee of Genentech, Inc. N. Collinson is an employee of Roche Products, Ltd. S. Dimonaco is an employee of Roche Products, Ltd. J.C. Wilson, S. Jick and C. Meier have nothing to disclose.

Compliance with Ethics Guidelines. This article is based on previously conducted studies and does not contain any studies with human participants or animals performed by any of the authors. This study used only de-identified patient data; therefore, informed consent for this study was not needed.

Data Availability. The datasets analyzed in this study are available from the corresponding author (MarketScan data) and coauthors (CPRD data) on reasonable request.

Open Access. This article is distributed under the terms of the Creative Commons Attribution-NonCommercial 4.0 International License (http://creativecommons.org/licenses/ by-nc/4.0/), which permits any noncommercial use, distribution, and reproduction in any medium, provided you give appropriate credit to the original author(s) and the source, provide a link to the Creative Commons license, and indicate if changes were made.

\section{REFERENCES}

1. Mukhtyar C, Guillevin L, Cid MC, et al. EULAR recommendations for the management of large vessel vasculitis. Ann Rheum Dis. 2009;68:318-23.

2. Ness T, Bley TA, Schmidt WA, Lamprecht P. The diagnosis and treatment of giant cell arteritis. Dtsch Arztebl Int. 2013;110:376.

3. Ponte C, Rodrigues AF, O'Neill L, Luqmani RA. Giant cell arteritis: current treatment and management. World J Clin Cases. 2015;3:484-94.

4. Lawrence RC, Felson DT, Helmick CG, et al. Estimates of the prevalence of arthritis and other rheumatic conditions in the United States. Part II. Arthritis Rheum. 2008;58:26-35.

5. Hoffman GS. Giant cell arteritis. Ann Intern Med. 2016;165:ITC65-8.

6. Chandran AK, Udayakumar PD, Crowson CS, Warrington KJ, Matteson EL. The incidence of giant cell arteritis in Olmsted County, Minnesota, over a 60-year period 1950-2009. Scand J Rheumatol. 2015;44:215-8.

7. Ly KH, Regent A, Tamby MC, Mouthon L. Pathogenesis of giant cell arteritis: more than just an inflammatory condition? Autoimmun Rev. 2010;9:635-45.

8. Gabriel SE, Michaud K. Epidemiological studies in incidence, prevalence, mortality, and comorbidity of the rheumatic diseases. Arthritis Res Ther. 2009;11:229.

9. Hunder GG, Bloch DA, Michel BA, et al. The American College of Rheumatology 1990 criteria for the classification of giant cell arteritis. Arthritis Rheum. 1990;33:1122-8.

10. Salvarani C, Pipitone N, Versari A, Hunder GG. Clinical features of polymyalgia rheumatica and giant cell arteritis. Nat Rev Rheumatol. 2012;8:509-21.

11. Gonzalez-Gay MA, Castaneda S, Llorca J. Giant cell arteritis: visual loss is our major concern. J Rheumatol. 2016;43:1458-61.

12. Fraser JA, Weyand CM, Newman NJ, Biousse V. The treatment of giant cell arteritis. Rev Neurol Dis. 2008;5:140-52. 
13. Warrington KJ, Matteson EL. Management guidelines and outcome measures in giant cell arteritis (GCA). Clin Exp Rheumatol. 2007;25:137-41.

14. Dasgupta B, Borg FA, Hassan N, et al. BSR and BHPR guidelines for the management of giant cell arteritis. Rheumatology (Oxford). 2010;49:1594-7.

15. Dasgupta B, Giant Cell Arteritis Guideline Development Group. Concise guidance: diagnosis and management of giant cell arteritis. Clin Med (Lond). 2010;10:381-6.

16. Proven A, Gabriel SE, Orces C, O'Fallon WM, Hunder GG. Glucocorticoid therapy in giant cell arteritis: duration and adverse outcomes. Arthritis Rheum. 2003;49:703-8.

17. Andersson R, Malmvall BE, Bengtsson BA. Longterm corticosteroid treatment in giant cell arteritis. Acta Med Scand. 1986;220:465-9.

18. Petri H, Nevitt A, Sarsour K, Napalkov P, Collinson N. Incidence of giant cell arteritis and characteristics of patients: data-driven analysis of comorbidities. Arthritis Care Res (Hoboken). 2015;67:390-5.

19. Durand M, Thomas SL. Incidence of infections in patients with giant cell arteritis: a cohort study. Arthritis Care Res (Hoboken). 2012;64:581-8.

20. Wilson JC, Sarsour K, Collinson N, et al. Serious adverse effects associated with glucocorticoid therapy in patients with giant cell arteritis (GCA): a nested case-control analysis. Semin Arthritis Rheum. 2017;46:819-27.

21. Tuckwell K, Collinson N, Dimonaco S, et al. Newly diagnosed vs. relapsing giant cell arteritis: baseline data from the GiACTA trial. Semin Arthritis Rheum. 2017;46:657-64.

22. De Vries F, Bracke M, Leufkens HG, Lammers JW, Cooper C, Van Staa TP. Fracture risk with intermittent high-dose oral glucocorticoid therapy. Arthritis Rheum. 2007;56:208-14.
23. Caplan L, Wolfe F, Russell AS, Michaud K. Corticosteroid use in rheumatoid arthritis: prevalence, predictors, correlates, and outcomes. J Rheumatol. 2007;34:696-705.

24. Miloslavsky EM, Naden RP, Bijlsma JW, et al. Development of a glucocorticoid toxicity index (GTI) using multicriteria decision analysis. Ann Rheum Dis. 2017;76:543-6.

25. Stone JH, Tuckwell K, Dimonaco S, et al. Trial of tocilizumab in giant-cell arteritis. $\mathrm{N}$ Engl J Med. 2017;377:317-28.

26. Herrett E, Gallagher AM, Bhaskaran K, et al. Data resource profile: Clinical Practice Research Datalink (CPRD). Int J Epidemiol. 2015;44:827-36.

27. Nguyen-Khoa BA, Goehring EL Jr, Alexander KA, Dong W, Napalkov P, Jones JK. Risk of significant infection in rheumatoid arthritis patients switching anti-tumor necrosis factor-alpha drugs. Semin Arthritis Rheum. 2012;42:119-26.

28. Salvarani C, Crowson CS, O'Fallon WM, Hunder GG, Gabriel SE. Reappraisal of the epidemiology of giant cell arteritis in Olmsted County, Minnesota, over a fifty-year period. Arthritis Rheum. 2004;51:264-8.

29. Smith CA, Fidler WJ, Pinals RS. The epidemiology of giant cell arteritis. Report of a ten-year study in Shelby County, Tennessee. Arthritis Rheum. 1983;26:1214-9.

30. Broder MS, Sarsour K, Chang E, et al. Corticosteroid-related adverse events in patients with giant cell arteritis: a claims-based analysis. Semin Arthritis Rheum. 2016;46:246-52.

31. Schmidt J, Smail A, Roche B, et al. Incidence of severe infections and infection-related mortality during the course of giant cell arteritis: a multicenter, prospective, double-cohort study. Arthritis Rheumatol. 2016;68:1477-82. 